\title{
Extraction of genomic DNA from yeasts for PCR-based applications
}

\section{Protocol For: \\ Extraction of genomic DNA from yeasts for PCR-based applications}

Marko Lõoke, Kersti Kristjuhan, and Arnold Kristjuhan

Institute of Molecular and Cell Biology, University of Tartu, Tartu, Estonia

BioTechniques Protocol Guide, January 2017; doi 10.2144/000114497

See full protocol online: http://www.biotechniques.com/BiotechniquesJournal/2011/May/Extraction-of-genomic-DNA-from-yeasts-for-PCR-basedapplications/biotechniques-315236.html

Protocol Summary: We have developed a quick and low-cost genomic DNA extraction protocol from yeast cells for PCR-based applications. This method does not require any enzymes, hazardous chemicals, or extreme temperatures, and is especially powerful for simultaneous analysis of a large number of samples. DNA can be efficiently extracted from different yeast species (Kluyveromyces lactis, Hansenula polymorpha, Schizosaccharomyces pombe, Candida albicans, Pichia pastoris, and Saccharomyces cerevisiae). The protocol involves lysis of yeast colonies or cells from liquid culture in a lithium acetate (LiOAc)-SDS solution and subsequent precipitation of DNA with ethanol. Approximately 100 nanograms of total genomic DNA can be extracted from $1 \times 107$ cells. DNA extracted by this method is suitable for a variety of PCR-based applications (including colony PCR, real-time qPCR, and DNA sequencing) for amplification of DNA fragments of $\leq 3500 \mathrm{bp}$.
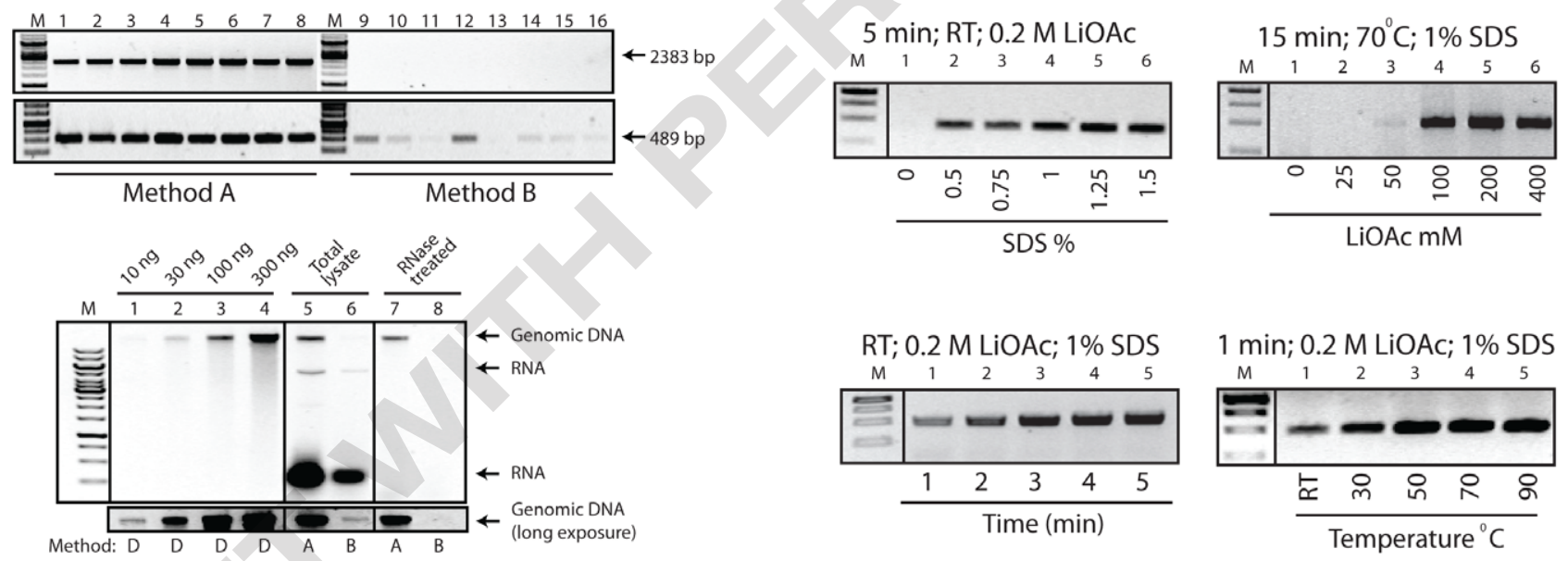

Protocol Schematic: Optimization of LiOAc-SDS lysis in S. cerevisiae. (A) PCR amplification of S. cerevisiae gDNA prepared from single yeast colonies by LiOAc-SDS (Method A; lanes 1-8) or by SDS treatment (Method B; lanes 9-16). gDNA fragments (489 bp and 2383 bp) from VPS13 locus were amplified with FirePol Taq polymerase (Solis BioDyne, Tartu, Estonia) for 30 cycles $\left(95^{\circ} \mathrm{C}\right.$ for $15 \mathrm{~s}, 58^{\circ} \mathrm{C}$ for $30 \mathrm{~s}$, and $72^{\circ} \mathrm{C}$ for $\left.150 \mathrm{~s}\right)$. The reaction buffer was supplemented with $1 \%$ Triton $\mathrm{X}-100$ for samples 9-16 (as recommended in Reference 4). (B) Total lysate of $1 \times 107$ cells prepared by Methods A and B was analyzed on a 0.9\% TAE-agarose gel. Lanes 1-4: dilution series of yeast gDNA concentration standards prepared by Method D; lane 5: gDNA prepared by Method A; lane 6: gDNA prepared by Method B; lanes 7-8: gDNA prepared by Methods A and B were purified further by RNase $\mathrm{A}$ and proteinase $\mathrm{K}$ treatments, followed by phenol:chloroform extraction and ethanol precipitation of gDNA. A longer exposure of the gDNA bands is shown underneath the main gel. (C-F) Optimization of LiOAc-SDS lysis protocol conditions. Concentrations of SDS and LiOAc, lysis time and temperature were tested (panels C, D, E, and F, respectively). Variable parameters are indicated below the panels and constant parameters are shown on top. gDNA fragment from VPS13 locus (489 bp) was amplified by PCR. RT, room temperature. See Figure 2B for DNA size marker (lanes M). PCR products were analyzed on 0.9\% TAE-agarose gel electrophoresis, stained with ethidium bromide, and photographed. 